\title{
Modeling Electronic-Nuclear Interactions for Excitation Energy Transfer Processes in Light-Harvesting Complexes
}

\author{
Mi Kyung Lee* and David F. Coker* \\ Department of Chemistry, Boston University, 590 Commonwealth Avenue, Boston, MA \\ 02215, USA
}

E-mail: mlee03@bu.edu; coker@bu.edu

\section{Simple analytic model for MM correlation function cal- culation of $J_{\mathrm{MM}}^{(\alpha)}(\omega)$}

The standard approach, based on the classical trajectories from an equilibrated MD simulation using a generalized ground state MM force field, has been employed to calculate site dependent spectral densities, $J^{(\alpha)}(\omega)$, for a variety of light-harvesting complexes. ${ }^{1-5}$ The time-dependent excitation energy fluctuations are computed for the configurations along the trajectory and the excitation energy correlation function is averaged over different trajectory segments assuming ergodicity. This ubiquitous method also assumes that the MD surface determining the component frequencies in the correlation function resembles the QM ground state surface from which the relative excitation energy fluctuation is computed. However, if the ground state QM and MM surfaces differ, especially for the modes that influence EET, the spectral densities computed from this standard approach may be characterized by peaks at spurious frequencies with unrealistic amplitudes. Here we outline a simple analyt- 
ical model that we use to understand how the spectral densities change as a result of the mismatch in the MM and QM ground state surfaces.

In this analysis, the ground state, $V_{\mathrm{g}}^{(\alpha)}\left(\mathbf{Q}^{(\alpha)}-\mathbf{Q}_{\mathrm{g}}^{(\alpha)}\right)$, and excited state surfaces, $V_{\mathrm{e}}^{(\alpha)}\left(\mathbf{Q}^{(\alpha)}-\right.$ $\mathrm{Q}_{\mathrm{e}}^{(\alpha)}$ ), of chromophore $\alpha$ are functions of a common set of bath normal mode coordinates $\mathbf{Q}^{(\alpha)}$, which, in the ground state, oscillate around the equilibrium point $\mathbf{Q}_{\mathrm{g}}^{(\alpha)}$, and around $\mathrm{Q}_{\mathrm{e}}^{(\alpha)}$ in the excited state. We further assume the QM surfaces, $V_{\mathrm{g}}^{(\alpha)}$ and $V_{\mathrm{e}}^{(\alpha)}$, are locally harmonic in these coordinates, and that they have the same curvature characterized by normal mode frequencies of $\omega_{i}^{(\alpha)}$. In general, this a reasonable approximation, the reliability of which has been explored in studies comparing ${ }^{6}$ difference fluorescence line narrowing ${ }^{7-9}$ and hole burning spectra. ${ }^{10}$ On the other hand, the model MM potential surface, $V_{\mathrm{MM}}^{(\alpha)}\left(\mathbf{q}^{(\alpha)}-\mathbf{q}_{\mathrm{MM}}^{(\alpha)}\right)$, in general, may have different frequencies, $\Omega_{i}^{(\alpha)}$ and even different normal modes, $\mathbf{q}^{(\alpha)}$, rotated relative to $\mathbf{Q}^{(\alpha)}$, and they may describe motion oscillating around a different MM equilibrium point $\mathbf{q}_{\mathrm{MM}}^{(\alpha)}$. Thus, in our model of the MM excitation energy correlation function calculation we assume that the relevant potential surfaces are:

$$
\begin{gathered}
V_{\mathrm{g}}^{(\alpha)}\left(\mathbf{Q}^{(\alpha)}\right)=\frac{1}{2} \sum_{i} \omega_{i}^{(\alpha) 2}\left(Q_{i}^{(\alpha)}-Q_{\mathrm{g} i}^{(\alpha)}\right)^{2} \\
V_{\mathrm{e}}^{(\alpha)}\left(\mathbf{Q}^{(\alpha)}\right)=\frac{1}{2} \sum_{i} \omega_{i}^{(\alpha) 2}\left(Q_{i}^{(\alpha)}-Q_{\mathrm{e} i}^{(\alpha)}\right)^{2}+E^{(\alpha)} \\
V_{\mathrm{MM}}^{(\alpha)}\left(\mathbf{q}^{(\alpha)}\right)=\frac{1}{2} \sum_{i} \Omega_{i}^{(\alpha) 2}\left(q_{i}^{(\alpha)}-q_{\mathrm{MM} i}^{(\alpha)}\right)^{2}+E_{\mathrm{MM}}^{(\alpha)}
\end{gathered}
$$

The key quantities governing the excited state relaxation after chromophore photoexcitation are the excited state reorganization energies, $\lambda_{i}^{(\alpha)}$. These values quantify the magnitude of the energy that chromophore $\alpha$ needs to dissipate in mode $i$ to relax from the non-equilibrium Franck-Condon point where the excited state energy is, $V_{\mathrm{e}}^{(\alpha)}\left(Q_{\mathrm{g} i}^{(\alpha)}-Q_{\mathrm{e} i}^{(\alpha)}\right)$, to the equilibrium point, $V_{\mathrm{e}}^{(\alpha)}\left(Q_{\mathrm{e} i}^{(\alpha)}\right)$. With this definition, it is readily shown that the reorganization energies for the different bath modes are simply related to the properties of the 
ground and excited state potentials as follows:

$$
\lambda_{i}^{(\alpha)}=\frac{1}{2} \omega_{i}^{(\alpha) 2}\left(Q_{\mathrm{e} i}^{(\alpha)}-Q_{\mathrm{g} i}^{(\alpha)}\right)^{2}=S_{i}^{(\alpha)} \hbar \omega_{i}^{(\alpha)}
$$

where we have defined the dimensionless Huang-Rhys factor, $S_{i}^{(\alpha)}$, as the number of vibrational quanta, $\hbar \omega_{i}^{(\alpha)}$, in the mode of interest that is equivalent to its reorganization energy. The total reorganization energy for all bath modes of chromophore $\alpha$ is then $\lambda^{(\alpha)}=\sum_{i} \lambda_{i}^{(\alpha)}$ and $S^{(\alpha)}=\sum_{i} S_{i}^{(\alpha)}$. With this model, all the parameters introduced in the main text - the excitation energy fluctuation, $\delta \epsilon^{(\alpha)}$, and the system-bath bi-linear coupling constants, $c_{i}^{(\alpha)}$, can now be identified:

$$
\delta \epsilon^{(\alpha)}\left(Q_{i}^{(\alpha)}\right)=V_{\mathrm{e}}^{(\alpha)}\left(Q_{i}^{(\alpha)}\right)-V_{\mathrm{g}}^{(\alpha)}\left(Q_{i}^{(\alpha)}\right)=\mathrm{constant}-\sum_{i} \omega_{i}^{(\alpha) 2}\left(Q_{\mathrm{e} i}^{(\alpha)}-Q_{\mathrm{g} i}^{(\alpha)}\right) Q_{i}^{(\alpha)},
$$

where the constant $=E^{(\alpha)}+\frac{1}{2} \sum_{i} \omega_{i}^{(\alpha)_{2}}\left(Q_{\mathrm{e} i}^{(\alpha)_{2}}-Q_{\mathrm{g} i}^{(\alpha)}\right)$, and the linear coupling constants defined above Equation (1) in the main text are related to the various model parameters as follows:

$$
\begin{aligned}
& c_{i}^{(\alpha)}=\omega_{i}^{(\alpha) 2}\left(Q_{\mathrm{e} i}^{(\alpha)}-Q_{\mathrm{g} i}^{(\alpha)}\right)=\sqrt{2 \lambda_{i}^{(\alpha)}} \omega_{i}^{(\alpha)}=\sqrt{2 \hbar \omega_{i}^{(\alpha)} S_{i}^{(\alpha)}} \omega_{i}^{(\alpha)} \\
& \lambda_{i}^{(\alpha)}=c_{i}^{(\alpha) 2} / 2 \omega_{i}^{(\alpha) 2}
\end{aligned}
$$

Giving the spectral density defined in Equation (2) of the main text as:

$$
J^{(\alpha)}(\omega)=\pi \sum_{i} \omega_{i}^{(\alpha)} \lambda_{i}^{(\alpha)} \delta\left(\omega-\omega_{i}^{(\alpha)}\right)
$$

We suppose that the QM normal mode coordinates obtained from the ground state electronic structure calculations are related to the MM normal modes by a linear transformation 
that has the form of a Duschinsky-like rotation,

$$
\mathbf{Q}^{(\alpha)}=\underline{\underline{D}}^{(\alpha)} \mathbf{q}^{(\alpha)}
$$

where the transformation matrix elements are $D_{k i}^{(\alpha)}=\hat{\mathbf{q}}_{i}^{(\alpha)}{ }^{T} \hat{\mathbf{Q}}_{k}^{(\alpha)}$ and the "hats" indicate unit vectors in the $3 N-6$ dimensional cartesian space describing chromophore $\alpha$ 's vibrations. Further, the MD trajectory obtained with the MM normal mode hamiltonian $H_{\mathrm{MM}}\left(\mathbf{p}^{(\alpha)}, \mathbf{q}^{(\alpha)}\right)=\frac{1}{2} \sum_{i} p_{i}^{(\alpha) 2}+V_{\mathrm{MM}}^{(\alpha)}\left(\mathbf{q}^{(\alpha)}\right)$, and that underlies our model correlation function calculation, has the form

$$
q_{i}^{(\alpha)}(t)=q_{\mathrm{MM} i}^{(\alpha)}+\left(q_{i}^{(\alpha)}(0)-q_{\mathrm{MM} i}^{(\alpha)}\right) \cos \Omega_{i}^{(\alpha)} t+\frac{p_{i}^{(\alpha)}(0)}{\Omega_{i}^{(\alpha)}} \sin \Omega_{i}^{(\alpha)} t
$$

and $\dot{q}_{i}^{(\alpha)}(t)=p_{i}^{(\alpha)}(t)$

The excitation energy, $\delta \epsilon^{(\alpha)}(t)$, that we need to compute along this trajectory, however, is the energy gap between the ground and excited QM surfaces i.e. $\delta \epsilon^{(\alpha)}(t)=\left[V_{\mathrm{e}}^{(\alpha)}\left(\mathbf{Q}^{(\alpha)}\left(\mathbf{q}^{(\alpha)}(t)\right)-\right.\right.$ $V_{\mathrm{g}}^{(\alpha)}\left(\mathbf{Q}^{(\alpha)}\left(\mathbf{q}^{(\alpha)}(t)\right)\right]$ and the required correlation function is $C_{\mathrm{MM}}^{(\alpha)}(t)=\left\langle\delta \epsilon^{(\alpha)}(0) \delta \epsilon^{(\alpha)}(t)\right\rangle_{\mathrm{MM}}$, where the thermal MM average indicated by $\langle\cdot\rangle_{\mathrm{MM}}$ is obtained by integrating over MD trajectory initial conditions according to the following result:

$$
C_{\mathrm{MM}}^{(\alpha)}(t)=\frac{1}{Z_{\mathrm{MM}}^{(\alpha)}} \int d \mathbf{p}^{(\alpha)}(0) \int d \mathbf{q}^{(\alpha)}(0) \exp \left[-\beta H_{\mathrm{MM}}^{(\alpha)}\left(\mathbf{p}^{(\alpha)}(0), \mathbf{q}^{(\alpha)}(0)\right)\right] \delta \epsilon^{(\alpha)}(0) \delta \epsilon^{(\alpha)}(t)
$$

with $Z_{\mathrm{MM}}^{(\alpha)}=\int d \mathbf{p}^{(\alpha)}(0) \int d \mathbf{q}^{(\alpha)}(0) \exp \left[-\beta H_{\mathrm{MM}}^{(\alpha)}\left(\mathbf{p}^{(\alpha)}(0), \mathbf{q}^{(\alpha)}(0)\right)\right]$.

The energy gap term, $\delta \epsilon^{(\alpha)}(0) \delta \epsilon^{(\alpha)}(t)$, in the above integral is given in terms of the QM normal modes but the trajectory and the integration over initial conditions are written in terms of MM coordinates so we must use the transformation in Equation (S8) to evaluate this quantity. The gaussian integrals in the expression that results after these manipulations can be performed analytically and the time dependent part of the correlation function needed 
for the spectral density computation with this model is obtained as

$$
C_{\mathrm{MM}}^{(\alpha)}(t)=\frac{2}{\beta} \sum_{i} \sum_{k}\left(\frac{\omega_{k}^{(\alpha)} D_{k i}^{(\alpha)}}{\Omega_{i}^{(\alpha)}}\right)^{2} \lambda_{k}^{(\alpha)} \cos \Omega_{i}^{(\alpha)} t
$$

Transforming as outlined below Equation (2) of the main text we obtain the model MM spectral density as

$$
J_{\mathrm{MM}}^{(\alpha)}(\omega)=\pi \omega \sum_{i} \sum_{k}\left(\frac{\omega_{k}^{(\alpha)} D_{k i}^{(\alpha)}}{\Omega_{i}^{(\alpha)}}\right)^{2} \lambda_{k}^{(\alpha)} \delta\left(\omega-\Omega_{i}^{(\alpha)}\right)
$$

Comparing this result with the definition of the spectral density in Equation (S7) we see that the peak frequencies computed using the MM model correlation function occur at the normal mode frequencies of the MM surface, $\Omega_{i}^{(\alpha)}$, which in general are different from the QM mode frequencies, $\omega_{i}^{(\alpha)}$, that govern where the peaks in the actual spectral density occur as given in Equation (S7). Further, the amplitudes of the peaks computed using Equation (S12), are not simply proportional to $\omega_{i}^{(\alpha)} \lambda_{i}^{(\alpha)}$ as in Equation (S7), rather they are linear combinations of the QM reorganization energies in which the combination coefficients are determined by how the MM surface normal modes are rotated from the QM normal modes and the ratio of their frequencies, i.e. the magnitude of the model MM spectral density peak at frequency $\Omega_{i}^{(\alpha)}$ is $\Omega_{i}^{(\alpha)} \sum_{k} \lambda_{k}^{(\alpha)}\left(D_{k i}^{(\alpha)} \omega_{k}^{(\alpha)} / \Omega_{i}^{(\alpha)}\right)^{2}$.

With a general purpose MM potential surface the spectral densities obtained from an MM trajectory correlation function analysis can thus be significantly different from the true spectral density due to the differences in the shape of the MM model surface compared to the true QM potentials. This can lead to a strong dependence of the spectral density computed in this way on the the choice of MM force field. ${ }^{11-13}$ 


\section{Computational details}

Initial configurations of the BChls for the two-layer $\mathrm{ONIOM}^{14}$ geometry optimization with electronic embedding as implemented in Gaussian ${ }^{15}$ were randomly selected from the Molecular Dynamics simulation that is detailed in the SI of reference. ${ }^{16}$ For the high (QM) ONIOM layer, the total number of BChl atoms was reduced from 140 to 85 atoms by truncating the BChl at the phytyl tail with a hydrogen linking atom. The low ONIOM layer was parameterized with the CHARMM force field used in the dynamics, and consisted of the truncated phytyl tail, all protein and non-QM BChl residues of the FMO trimer, as well as all water molecules within $10 \AA$ of the center of mass of the QM BChl. For each BChl site, a total of 255 normal modes were calculated after the geometry optimization and the resulting frequencies were scaled uniformly by factors ranging from 0.95 to 0.97 for the various functionals to optimally reproduce experimental frequencies. The frequencies for the six lowest modes for each chromophore were non-zero due to the inhomogeneous static electric field. Generally their magnitudes were less than 20-40 $\mathrm{cm}^{-1}$. The TDDFT excited state forces in cartesian coordinates were calculated analytically at the ground state optimized geometries. Rotating these vectors to mass-weighted Cartesian normal mode coordinates give the bi-linear cou-

pling constants, $c_{i}^{(\alpha)}$, described above Equation (1) in the main text. Pople's double-zeta basis set, 6-31G(d), was used with various DFT functionals (see details below) to describe the QM level.

\section{Results: Huang-Rhys Factors}

Using the normal mode procedure outlined in the main text and the computational details presented above, the HR factors were computed for each of the eight bacteriochlorophylls (BChls) in the three different subunits of the FMO trimer following local chromophore geometry optimizations from a single well equilibrated MD configuration, and these results are presented in Figure S1. In each panel of Figure S1, the three different sets of HR factors 


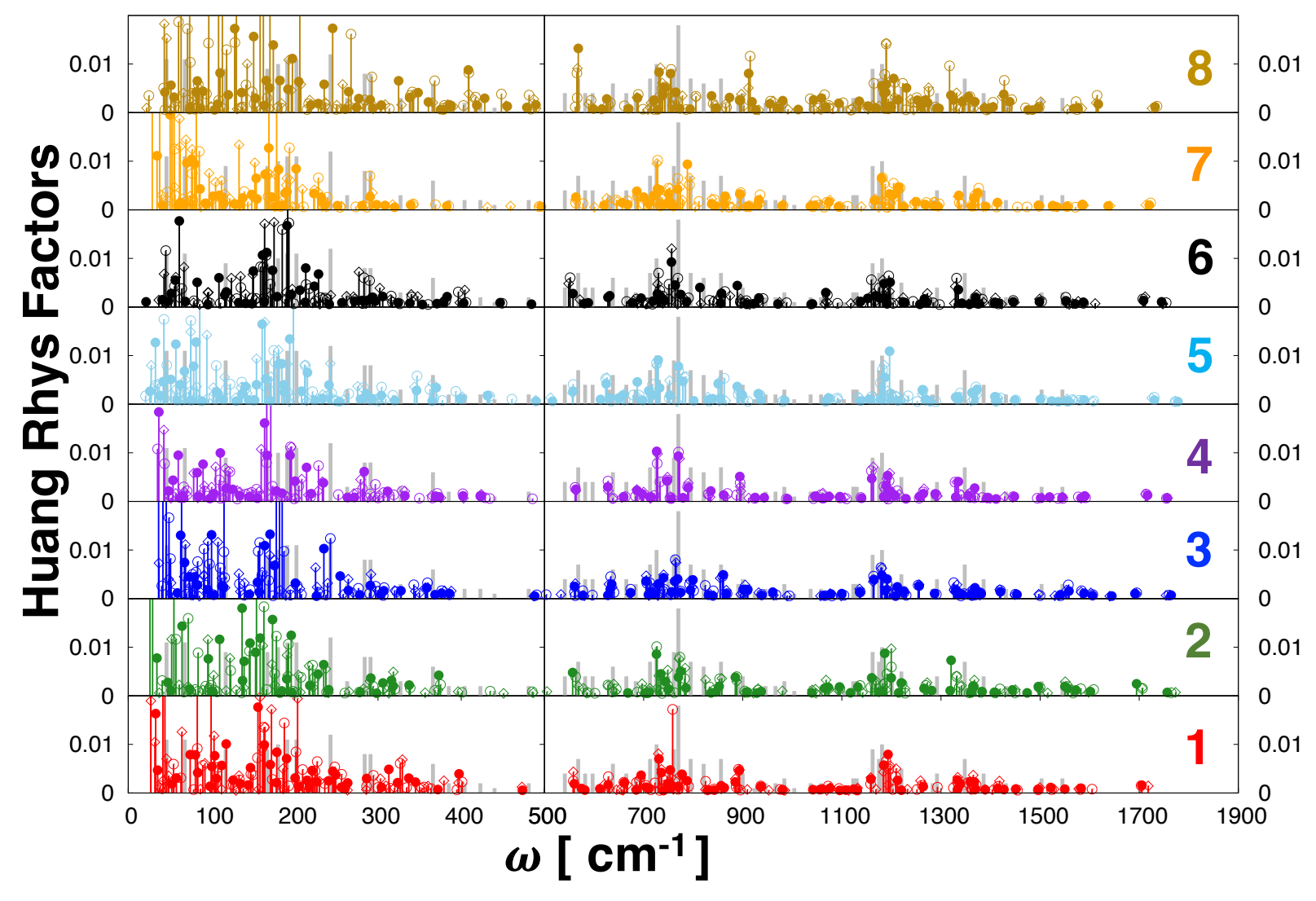

Figure S1: Comparison of experimental $\triangle \mathrm{FLN}$ HR factors (gray) with current calculated HR factors for BChl1 (red), BChl2 (blue), BChl3 (blue), BChl4 (purple), BChl5 (skyblue), BChl6 (thick-black), BChl7 (orange), and BChl8 (brown). For each of the eight sub-panels, HR factors for BChls 1-8 in FMO subunit A are shown in filled circles; subunit B in open circles; and subunit $\mathrm{C}$ in open diamonds. 
calculated for the BChls in the monomer subunits $\mathrm{A}, \mathrm{B}$, and $\mathrm{C}$ of the FMO trimer are plotted as filled circles, open circles, and open diamonds, respectively. These calculated results are compared to the experimental $\triangle \mathrm{FLN} H R$ factors (grey bars, signal dominated by BChl3). The general agreement between the calculated and experimental HR factors and their vibrational mode frequencies is very good, and the differences from chromophore to chromophore and subunit to subunit give an indication of the dispersion estimated in these quantities associated with the different local structures sampled from the room temperature thermal distribution of the protein.

\section{Results: Site-dependent spectral densities}

For each panel in Figure S2, the color coded curves give our calculated spectral densities for the BChls in the different subunits (different line types) of the FMO trimer. These results are compared with other representative work: the thin-black curves correspond to spectral density calculations obtained by computing ZINDO/S excitation energy fluctuations along the CHARMM ground state surface, ${ }^{12,13}$ and the thick-black curves give results for BChl1 and $\mathrm{BChl}^{3}$ computed using (TD)BLYP/3-21G excitation energies computed along an Amber MM trajectory. In addition, these computational results are compared to two different experimentally determined spectral densities: the gray filled curve gives the spectral density computed from the reported experimental $\triangle \mathrm{FLN}$ HR factors, ${ }^{7}$ which have been Gaussian broadened in exactly the same manner as our current calculated results as detailed in the main text. Finally, the dark-pink curve reported in the low-frequency panels corresponds to the analytic form of the spectral density ${ }^{9}$ that has been fit to the FLN measurements. ${ }^{8} 9$ The similarity in shape and magnitude of the two experimental spectral densities rationalizes the estimated broadening we have employed to model the experimental spectral densities from the HR factors. 


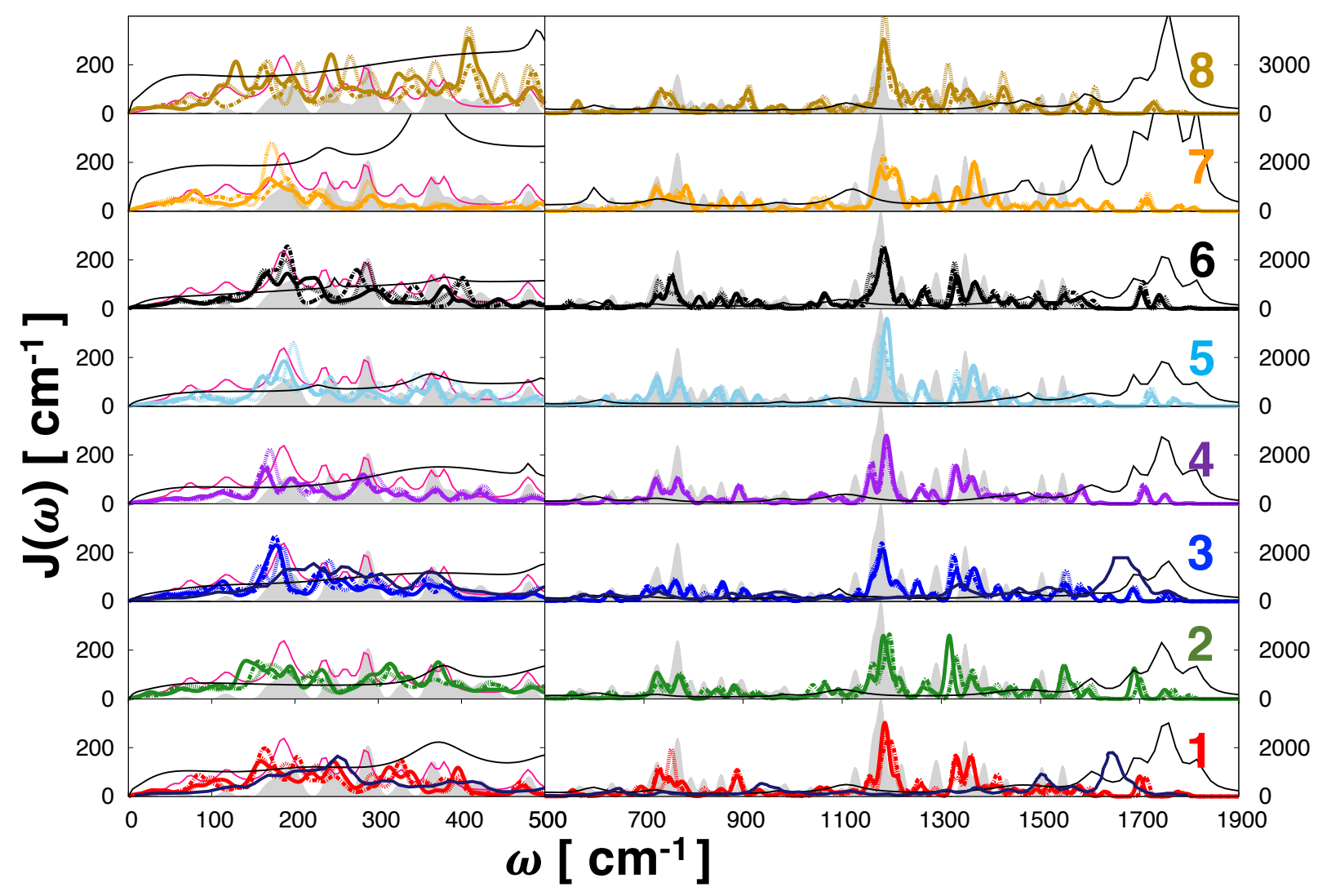

Figure S2: Comparisons of (1) gaussian broadened intra-chromophore spectral density extracted from $\triangle \mathrm{FLN}$ experiments ${ }^{7}$ in light-gray filled curve; (2) thin dark-pink low-frequency spectral density from FLN experimental data; ${ }^{8,9}(3)$ current $J(\omega)=J^{\mathrm{vib}}(\omega)+J^{\text {inter }}(\omega)$ for BChl1 (red), BChl2 (green), BChl3 (blue), BChl4 (purple), BChl5 (skyblue), BChl6 (black), BChl7 (orange), and BChl8 (brown) with the thick solid lines for FMO subunit A, dashed for FMO subunit B, and dot-dashed for FMO subunit C; (4) spectral densities for BChls 1-8 calculated with ZINDO/S/CHARMM from the Supporting Information of ${ }^{12,13}$ shown with thin black lines; and (5) spectral densities with at $77 \mathrm{~K}$ for BChl1 and BChl3 from ${ }^{3}$ shown with thick dark blue lines. 


\section{Results: Cumulative coupling strengths}

Comparisons of various estimates and different components of spectral densities for different sites are illustrated in Figure S3. In this figure, the normalized cumulative coupling strength distributions, $C(\omega)=\int_{0}^{\omega} d \omega^{\prime} J_{\alpha}\left(\omega^{\prime}\right) / \omega^{\prime} /\left(\pi \lambda_{\alpha}\right)$, for the computed (in colored solid lines) and experimental spectral densities (in gray) are presented. The trimodal distributions of reorganization energies across the entire frequency range are very similar for the eight chromophores and they closely resemble the experimental cumulative distribution. The total reorganization energies of the individual chromophores are listed in the lower-right. BChl8 has the largest reorganization energy of $240.8 \mathrm{~cm}^{-1}$, while those of BChls 1 to 7 ranges from 134 to $150 \mathrm{~cm}^{-1}$.

\section{Results: BChl monomer studies}

In Figure S4 we present results for the computed intramolecular spectral density of a gas phase bacteriochlorophyll molecule analogous to those presented in Figure 2 of the main text only here the electronic structure method used is the semi-empirical ZINDO/S approach. Comparing the results in panel (b) of this figure with the density functional results (TDB3LYP/3-21G) displayed in Figure 2 of the main text (reproduced in panel (a) here), we see that the ZINDO/S approach provides unreliable estimates of vibrational frequencies across the entire range resulting in a poor description of the spectral density as computed using excitation energy correlation function methods. ${ }^{12,13}$

\section{Study of influence of different functionals on computed spectral densities}

In Table S1, the Huang-Rhys factors and reorganization energies for the eight BChls obtained with eight different functionals are listed. The popular B3LYP and PBE0 are representa- 


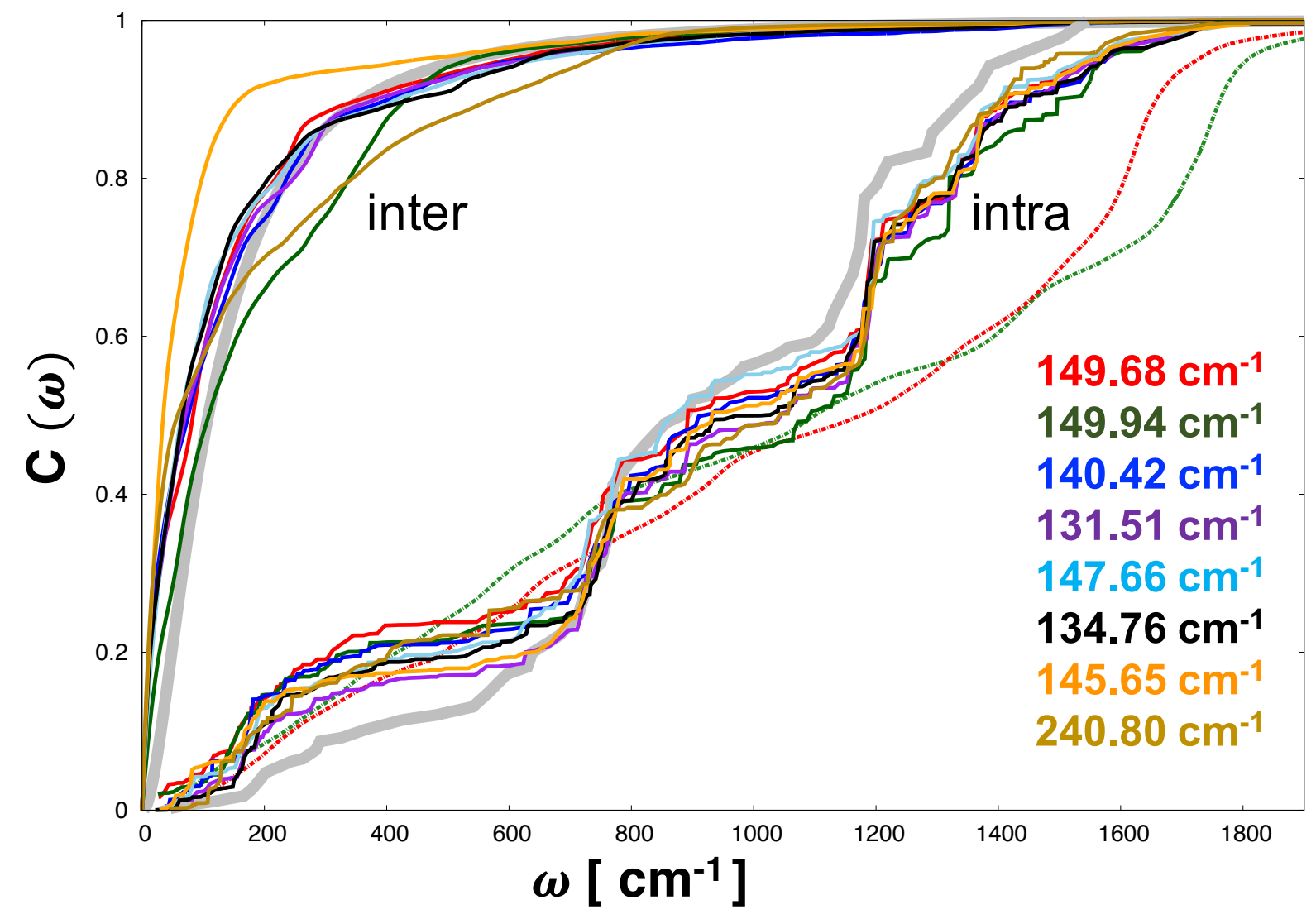

Figure S3: Normalized cumulative coupling strength distribution $C(\omega)=$ $\int_{0}^{\omega} d \omega^{\prime} J_{\alpha}\left(\omega^{\prime}\right) / \omega^{\prime} /\left(\pi \lambda_{\alpha}\right)$ for each of the intermolecular and intra-chromophore components of the environmental response for each of the chromophores in subunit A of the FMO trimer. The experimental results are shown in grey. The intermolecular spectral density results obtained from our long-range excitation energy correlation function calculations using the CHARMM force field give reorganization energies that range from $\sim 10 \mathrm{~cm}^{-1}$ to $\sim 40 \mathrm{~cm}^{-1}$ as reported in our previous work. ${ }^{16}$ The intra-chromophore results obtained from our PBE0 spectral densities are presented using the same color scheme as employed throughout the paper BChl1:red, BChl2:green, BChl3: blue, BChl4: purple, BChl5:cyan, BChl6: black, BChl4: orange, BClh8:gold. The computed intra-chromophore reorganization energies are shown in the insert and vary between $\sim 140 \mathrm{~cm}^{-1}$ and $\sim 240 \mathrm{~cm}^{-1}$ in excellent agreement with the experimental estimate $\left(\sim 210 \mathrm{~cm}^{-1}\right)$. The three steps in the experimental intra-chromophore cumulative distribution arising from the three important bands of modes in the spectral densities are well reproduced by our calculation results. The dashed red and green curves show cumulative spectral densities obtained using the excitation energy correlation function employing the Amber and CHARMM force fields respectively. ${ }^{13}$ These curves fail to capture the basic three band structure of the spectral density and the reorganization energies predicted by this approach are overestimated at more than $700 \mathrm{~cm}^{-1}$ and $1000 \mathrm{~cm}^{-1}$ respectively. 


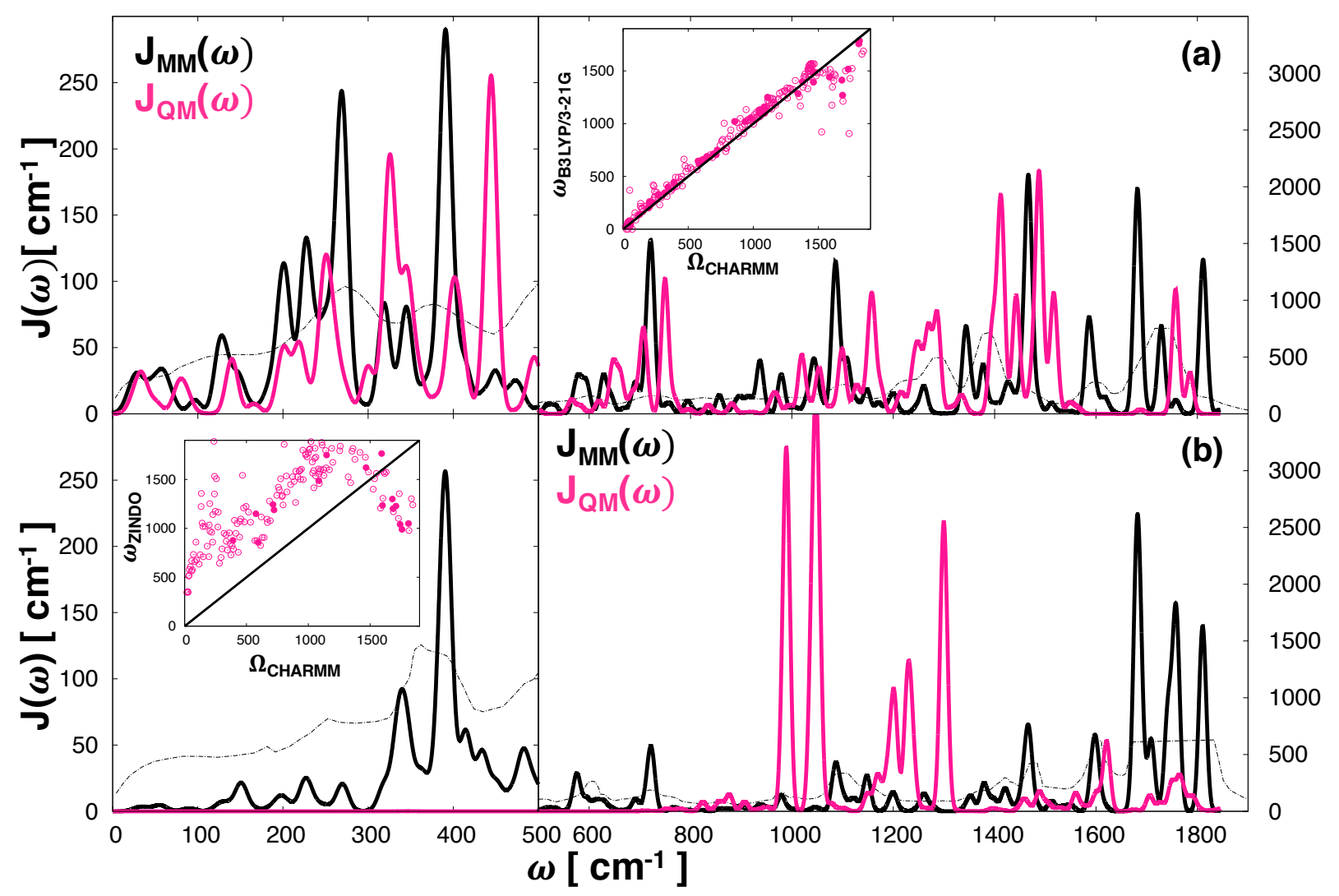

Figure S4: Comparison of spectral densities calculated for gas phase BChl on MM (CHARMM) and QM ground state harmonic surfaces. In panel (b) the spectral densities calculated with ZINDO/S with Equation (S7) (dark-pink) and Equation (S12) (black) are presented. The insert shows the correlation of the QM surface vibrational frequency, estimated using finite difference, with MM surface normal mode vibrational frequency. The dark-pink filled circles indicate modes with significant excitonic-vibrational coupling, i.e. reorganization energies larger than $1 \mathrm{~cm}^{-1}$. Panel (a) reproduce the TD B3LYP results detailed in Figure 2 of the main text for comparison. The dash-dot curves in each panel reproduce the average pigment-protein spectral densities in FMO computed using correlation function methods. ${ }^{12}$

Table S1: Huang Rhys factors and reorganization energies (in parenthesis, $\mathrm{cm}^{-1}$ ) calculated with different functionals

\begin{tabular}{|ccccccccc}
\hline Functional & BCL1 & BCL2 & BCL3 & BCL4 & BCL5 & BCL6 & BCL7 & BCL8 \\
\hline B3LYP & $0.41(146.74)$ & $0.36(146.72)$ & $0.29(132.87)$ & $0.23(124.00)$ & $0.30(139.71)$ & $0.23(129.70)$ & $0.30(136.59)$ & $0.37(192.73)$ \\
LC-BLYP (0.40) & $6.99(4937.4)$ & $2.80(2079.0)$ & $8.07(6392.9)$ & $9.73(7833.2)$ & $10.38(7547.2)$ & $7.15(5284.1)$ & $11.59(7930.1)$ & $2.39(1982.9)$ \\
LC-BLYP (0.18) & $0.26(147.92)$ & $0.19(221.92)$ & $0.22(144.51)$ & $0.20(129.37)$ & $0.22(136.34)$ & $0.21(133.58)$ & $0.21(149.50)$ & $1.84(1226.23)$ \\
CAM-B3LYP (0.33) & $4.10(1284.0)$ & $4.51(1982.5)$ & $2.14(736.3)$ & $3.08(1160.2)$ & $0.60(243.00)$ & $0.83(281.14)$ & $0.49(221.88)$ & $5.01(3262.6)$ \\
CAM-B3LYP (0.20) & $0.45(178.34)$ & $1.33(421.98)$ & $0.55(208.52)$ & $0.23(136.30)$ & $0.34(179.33)$ & $0.31(156.60)$ & $0.44(205.85)$ & $2.08(1238.6)$ \\
PBE0 (FMO-A) & $0.37(145.53)$ & $0.33(145.68)$ & $0.35(147.13)$ & $0.26(131.513)$ & $0.31(144.70)$ & $0.27(143.22)$ & $0.33(145.80)$ & $0.47(232.30)$ \\
PBE0 (FMO-B) & $0.35(147.13)$ & $0.28(147.36)$ & $0.32(147.11)$ & $0.25(133.69)$ & $0.29(138.93)$ & $0.28(140.18)$ & $0.33(145.94)$ & $0.40(184.25)$ \\
PBE0 (FMO-C) & $0.31(139.79)$ & $0.31(139.75)$ & $0.39(153.85)$ & $0.26(137.04)$ & $0.31(147.51)$ & $0.26(143.22)$ & $0.37(149.57)$ & $0.54(271.84)$ \\
HSE & $0.46(147.31)$ & $0.39(148.44)$ & $0.32(137.04)$ & $0.26(130.00)$ & $0.32(144.06)$ & $0.25(132.31)$ & $0.33(141.24)$ & $0.38(189.52)$ \\
HISS & $0.50(164.90)$ & $0.45(162.29)$ & $0.37(155.21)$ & $0.29(143.56)$ & $0.33(148.99)$ & $0.30(145.92)$ & $0.38(160.00)$ & $0.67(325.99)$ \\
\hline
\end{tabular}


tive hybrid functionals with roughly the same percentage of exact HF exchange (20-25\%) to approximately incorporate nonlocal exchange effects. For each of the range-separated functionals LC-BLYP and CAM-B3LYP, results for two different range-separation parameters, $\omega$, are compared: the default range-separation parameters are $0.40 a_{0}^{-1}$ and $0.33 a_{0}^{-1}$ respectively, and the optimized $\omega$ values $\operatorname{are}^{17} 0.18 a_{0}^{-1}$ and $0.20 a_{0}^{-1}$. In addition, two less conventional functionals for these applications were tested in our studies: the HSE functional, unlike LC-BLYP and CAM-B3LYP, only incorporates HF exchange at short range and has been developed for periodic materials applications; the HISS functional, on the other hand, is parameterized with two separate range-separation parameter in order to only incorporate exact exchange in the mid-range region. Default range-separation parameters, as implemented in Gaussian 09, have been used. For all these different functionals, BChl8 consistently shows the largest reorganization energies. Generally, most functionals give reorganization energies for the other chromophores that are about half the magnitude of those predicted for BChl8.

Comparison of results calculated with different functionals also demonstrates the general insensitivity of the spectral densities computed with our approach to choice of electronic structure method. In Figure S5, the spectral densities calculated with the functionals summarized in Table S1 are presented for the different chromophores. For functionals with small amounts, or no exact exchange in the asymptotic limit, the differences in the spectral features for each BChl are small. However, as the percentage of exact exchange in the asymptotic limit increases (CAM-B3LYP and LC-BLYP), the calculated spectral densities differ not only when compared to other functionals, but also between different chromophores. In particular, the magnitudes of the spectral density peaks for BChl8 are significantly larger. Whether these differences are realistic or artifacts of using this functional needs further testing, for example, by optimally-tuning the range separation parameter. ${ }^{18}$ We speculate that the optimally tuned functionals will behave like PBE0, since the Mennucci group ${ }^{19}$ concluded that this functional best reproduces the electronic structure computed using high level correlated quantum chemistry methods in earlier benchmark studies. 


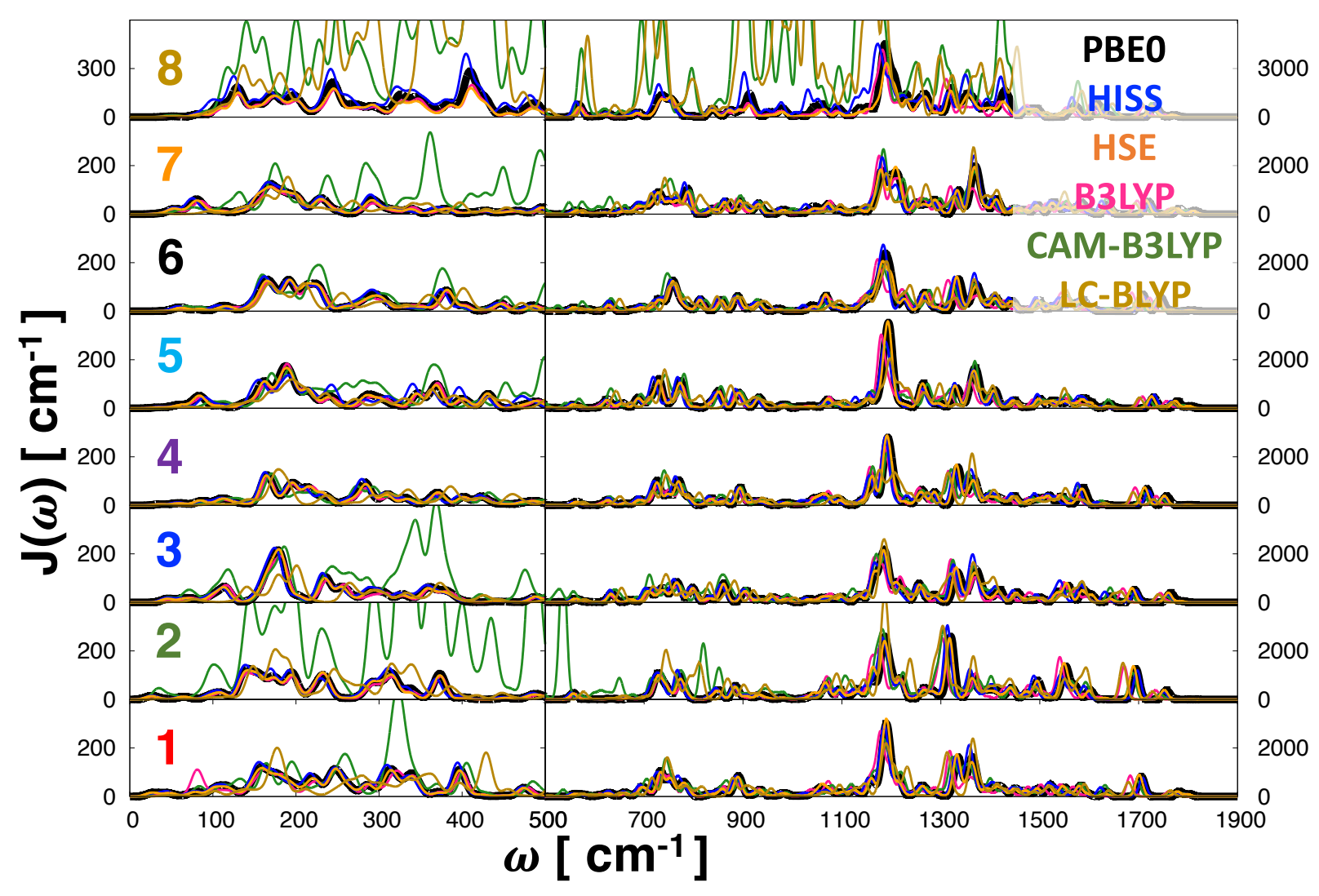

Figure S5: Comparisons of computed intra-chromophore spectral densities using different density functionals including: PBE0 (black), B3LYP (pink), CAM-B3LYP $[\omega=0.2]$ (green), HISS (blue), HSE (gold), LC-BLYP[ $\omega=0.18]$ (brown). Left panel presents low frequency results on an expanded scale, right panel shows high frequency results. 


\section{References}

(1) Damjanovic, A.; Kosztin, I.; Kleinekathöfer, U.; Schulten, K. Excitons in a Photosynthetic Light-Harvesting System: A Combined Molecular Dynamics, Quantum Chemistry, and Polaron Model Study. Phys. Rev. E 2002, 65, 031919-031943.

(2) Olbrich, C.; Strümpfer, J.; Schulten, K.; Kleinekathöfer, U. Theory and Simulation of the Environmental Effects on FMO Electronic Transitions. J. Phys. Chem. Lett. 2011, 2, 1771-1776.

(3) Valleau, S.; Eisfeld, A.; Aspuru-Guzik, A. On the Alternatives for Bath Correlators and Spectral Densities from Mixed Quantum-Classical Simulations. J. Chem. Phys. 2012, $13 \%, 224103-224116$.

(4) Aghtar, M.; Strümpfer, J.; Olbrich, C.; Schulten, K.; Kleinekathöfer, U. Different Types of Vibrations Interacting with Electronic Excitations in Phycoerythrin 545 and Fenna Matthews Olson Antenna Systems. J. Phys. Chem. Lett. 2014, 5, 3131-3137.

(5) Viani, L.; Corbella, M.; Curutchet, C.; O’Reilly, E. J.; Olaya-Castro, A.; Mennucci, B. Molecular Basis of the Exciton-Phonon Interactions in the PE545 Light-Harvesting Complex. Phys. Chem. Chem. Phys. 2014, 16, 16302-16311.

(6) Ratsep, M.; Cai, Z.; Reimers, J. R.; Freiberg, A. Demonstration and Interpretation of Significant Asymmetry in the Low-resolution and High-resolution Qy Fluorescence and Absorption Spectra of Bacteriochlorophyll A. J. Chem. Phys. 2011, 134, 024506024521.

(7) Ratsep, M.; Freiberg, A. Electron-Phonon and Vibronic Couplings in the FMO Bacteriochlorophyll A Antenna Complex Studied by Difference Fluorescence Line Narrowing. J. Lumin. 2007, 127, 251-259. 
(8) Wendling, M.; Pullerits, T.; Przyjalgowski, M. A.; Vulto, S. I. E.; Aartsma, T. J.; van Grondelle, R.; van Amerongen, H. Electron-Vibrational Coupling in the FennaMatthews-Olson Complex of Prosthecochloris aestuarii Determined by TemperatureDependent Absorption and Fluorescence Line-Narrowing Measurements. J. Phys. Chem. B 2000, 104, 5825-5831.

(9) Kreisbeck, C.; Kramer, T. Long-Lived Electronic Coherence in Dissipative Exciton Dynamics of Light-Harvesting Complexes. J. Phys. Chem. Lett. 2012, 3, 2828-2833.

(10) Kell, A.; Feng, X.; Reppert, M.; Jankowiak, R. On the Shape of the Phonon Spectral Density in Photosynthetic Complexes. J. Phys. Chem. B 2013, 117, 7317-7323.

(11) Kim, C. W.; Park, J. W.; Rhee, Y. M. Effect of Chromophore Potential Model on the Description of Exciton-Phonon Interactions. J. Phys. Chem. Letts. 2015, 6, 2875-2880.

(12) Chandrasekaran, S.; Aghtar, M.; Valleau, S.; Aspuru-Guzik, A.; Kleinekathöfer, U. Comparison between CHARMM \& AMBER and ZINDO vs. TDDFT. J. Phys. Chem. $B$ 2015, 119, 9995-10004.

(13) Wang, X.; Ritschel, G.; Wuster, S.; Eisfeld, A. Open Quantum System Parameters for Light-Harvesting Complexes from Molecular Dynamics. Phys. Chem. Chem. Phys. 2015, 17, 25629-25641.

(14) Vreven, T.; Morokuma, K. Hybrid Methods:ONIOM(QM:MM) and QM/MM. Ann. Rep. Comp. Chem. 2006, 2, 35-49.

(15) Frisch, M. J. et al. Gaussian 09, Revision B.01. Gaussian, Inc.: Wallingford, CT, 2010.

(16) Rivera, E.; Montemayor, D.; Masia, M.; Coker, D. F. Influence of Site-Dependent Pigment-Protein Interactions on Excitation Energy Transfer in Photosynthetic Light Harvesting. J. Phys. Chem. B 2013, 117, 5510-5521. 
(17) Higashi, M.; Kosugi, T.; Hayashi, S.; Saito, S. Theoretical Study on Excited States of Bacteriochlorophyll A in Solutions with Density Functional Assessment. J. Phys. Chem. B 2014, 118, 10906-10918.

(18) Refaely-Abramson, S.; Jain, M.; Sharifzadeh, S.; Neaton, J. B.; Kronik, L. Solid-State Optical Absorption from Optimally Tuned Time-Dependent Range-separated Hybrid Density Functional Theory. Phys. Rev. B 2015, 92, 081204-081210.

(19) List, N. H.; Curutchet, C.; Knecht, S.; Mennucci, B.; Kongsted, J. Toward Reliable Prediction of the Energy Ladder in Multichromophoric Systems: A Benchmark Study on the FMO Light-Harvesting Complex. J. Chem. Theo. Comput. 2013, 9, 4928-4938. 